\title{
Educación para la paz: cuestiones y retos para la Escuela
}

\section{Education for peace: issues and challenges for Schools}

\author{
Vanessa Cuenca Rivas \\ Maestrante en Educación y Cultura de Paz. Universidad Surcolombiana. \\ Neiva. Colombia. \\ vanessacuenca1027@gmail.com
}

\section{Resumen}

En el presente documento se tiene como objeto dialogar sobre algunas cuestiones y retos que la educación para la paz, le plantea a la escuela en el contexto Colombiano. A partir un esbozo sobre qué se entiende por educación para la paz, los paradigmas en educación para la paz desde los planteamientos del docente español Xesus Jares, la educación para la paz en Colombia en donde se hace mención a la normatividad en torno a los temas de paz para el contexto educativo, referenciando especialmente la cátedra para la paz adoptada mediante la ley 1732 del 1 de septiembre de 2014. Para finalmente develar algunos retos para el desarrollo de la educación para la paz en la escuela, como parte del proceso de construcción de una cultura de paz desde el rescate de la educación como medio ético- político.

Palabras claves: Educación para la paz, cultura de paz, paradigmas.

\section{Abstract}

This document aims to discuss issues and challenges that education for peace causes in schools in Colombia. The document starts with a suggestion of what is understood by education for peace, followed by paradigms in education for peace from the proposal of the Spanish teacher Xesus Jares and education for peace in Colombia where mention is given to regulations regarding issues of peace in the educational context, making special reference to the Chair for peace adopted according to law 1732 of September 1, 2014. The document then reveals challenges faced by the development of education for peace in schools, as part of the process of building a culture of peace by improving education as an ethical-political means.

Keywords: Education for peace, culture of peace, paradigms.

\section{Introducción}

Los seres humanos como seres histórico-sociales, inacabados en permanente transformación siempre están creando categorías que les permitan comprender sus realidades $y$ al mismo tiempo tener una incidencia sobre las mismas. La paz y la violencia son categorías que las personas elaboran para ser y estar en el mundo, por lo tanto, el ser humano no es por naturaleza violento ni pacifico; sino que por el contrario el ser violento o pacifico es un aprendizaje social. De esta manera, si se comprende que la paz y la violencia son construcciones culturales, se puede decir que son sujetas a un aprender pero también a un desaprender. En tal sentido, es posible hablar de una educación para la paz, que fomente la construcción de una cultura de paz como proceso de desaprender la cultura de la violencia.

La educación para la paz es una realidad compleja, dinámica, que se construye y reinventa cotidianamente a través de la investigación, los aportes de diferentes escuelas pedagógicas y las experiencias cotidianas de trabajo de diferentes organizaciones sociales en torno a la pedagogía para la paz. Por tal razón, no es posible referenciar un concepto único sobre qué se entiende por educación para la paz; inclusive no hay conceptos absolutos sobre que es la paz. En tal sentido, la educación y la paz son dos campos que están en evolución permanente, en donde todo está por reinventarse y por lo tanto los modelos únicos y absolutos están en constante cuestionamiento. 
De esta manera, la educación para la paz implica varios retos para el contexto educativo formal; por que hablar de educación para la paz es poner en debate y reflexión crítica los modelos educativos, la concepción de ser humano y sociedad y el tipo de paz que se proyecta enseñar en la escuela.

\section{Paradigmas en la educación para la paz}

Los paradigmas sobre la educación para la paz están fundamentados en los sentidos, significados sobre paz y las concepciones de ser humano y sociedad que se estén construyendo en determinado contexto social y que direccionan la formas en que se ha entendido que debe ser una educación para la paz.

El español Xesus Jares hace mención a tres modelos generales en los cuales se ha entendido la educación para la paz: 1) el modelo Técnico positivista en donde se educa para la paz desde la paz negativa, entendida, como esa paz que privilegia la ausencia de guerras y conflictos sin importar los medios y fines que se utilicen. En tal sentido, se puede estar educando para la paz mediante prácticas fundamentadas en lógicas de violencia, evitándose con esto una reflexión crítica frente a esas lógicas y la justificación de la violencia como único medio para construir la paz.

Desde este paradigma de educación para la paz se proyecta como una forma de evitar la violencia directa y los conflictos, en tal sentido, los conflictos son vistos de forma negativa que atentan contra la armonía y equilibrio en el aula de clase. De esta manera, lo que se va a promover desde este paradigma es la formación de un estudiante pasivo, obediente y sin sentido de crítica frente a las realidades de su contexto social. Un estudiante que no va a poder encontrar oportunidades de aprendizaje en los conflictos en su escuela y vida cotidiana porque va a entender que estos son negativos y por lo tanto deben evitarse. Desconociéndose con esto que los conflictos hacen parte constitutiva de las relaciones humanas y potenciadores de la vida en sociedad. Y lo que debe formarse no es en su evitación sino en buscar estrategias creativas para su transformación adecuada en donde la violencia no sea una opción.

Por otro lado, desde el modelo positivista de la educación para la paz, esta se ve como una asignatura asilada de las otras y se privilegia la educación desde lo cognitivo, es decir, desde la transmisión de contenidos en donde la paz se convierte en un discurso que el estudiante debe aprender. 2) modelo Hermenéuticointerpretativo desde este modelo se fomenta la educación desde el reconocimiento del conflicto como parte de los aprendizajes de las personas, es por lo tanto, visto desde lo positivo. De igual forma, la educación para la paz que se contempla es una educación afectiva- relacional donde no se reduce la paz a una memorización de contenidos sino la paz como una dimensión viva, experiencial, que se asume como un estilo de vida, ligada a las realidades cotidianas y a las historias de vida de los estudiantes; lo que conlleva a que la educación tenga resonancia y sea significativa. 3) modelo socio-crítico en el marco de este modelo se resalta que la educación para la paz debe reivindicar el carácter político de la educación, es decir, asumir que la educación no es una acción neutra sino que indudablemente es una acción ético- política que está relacionada con la crítica reflexiva frente a las condiciones sociales, políticas, culturales y económicas de determinado territorio. En tal sentido, la educación para la paz no es vista como una materia más o transversal en el currículo, sino como una proyección hacia la vida de los estudiantes fuera del contexto escolar; para reflejarse en la cotidianidad de las relaciones con su familia, amistades y comunidad. Jares (citado por Jiménez, 2011) expone una comprensión de la educación para la paz desde el paradigma socio-critico como:

Un proceso educativo, dinámico, continuo y permanente, fundamentado en los conceptos de la paz positiva y en el conflicto, como elementos significantes y definidores, y que, a través de la aplicación de enfoques socioafectivos y problematizantes, pretende desarrollar un nuevo tipo de cultura, la cultura de la paz, que ayude a las personas a desvelar críticamente la realidad, para poder situarse ante ella, y actuar en consecuencia. Realidad que es compleja y conflictiva, y que la entendemos referida a la tres dimensiones relacionales en que se desenvuelve el ser humano: consigo mismo y con los demás; con y desde las interacciones y estructuras sociales por él creadas, y con el medio ambiente en el que transcurre la vida. En ese sentido hablamos de educación para la paz como encrucijada de una educación afectiva, una educación sociopolítica y una educación ambiental. (p. 22)

De esta manera, desde este enfoque se asume una educación para la paz desde los planteamientos del pedagogo brasileño Paulo Freire con su educación crítica, libertaria, problematizadora, afectiva, política y comprometida con las realidades de los contextos sociales.

Así pues, los paradigmas o modelos desde los cuales se ha proyectado la educación para la paz dan cuenta de las diferentes concepciones que se han elaborado sobre la misma y de como la educación para la paz no es un modelo educativo "universalista" que se puede imitar de un contexto a otro; sino que por el contrario la educación para la paz es una realidad situada en tiempo y lugar. Por eso resulta complejo pensarse en una educación para la paz homogenizadora.

Otro aspecto que nos permite develar los paradigmas de educación para la paz es que sin duda es que los horizontes de educación para la paz van muy ligados al tipo de sujeto de paz y sociedad que se representan los gobiernos e instituciones. 
Educación para la paz en Colombia: Desde la democracia, la convivencia y ciudadanía a la cátedra para la paz

El interés por la paz en el contexto educativo colombiano ha sido una constante, especialmente por los diferentes fenómenos de violencias que se han perpetuado de manera histórica no solo en la historia del país sino también en las historias de vida de un gran número de colombianos. Además, de una marcada desigualdad social, inequidad, injusticia y una permanente militarización de la vida cotidiana que conllevo a la formación de los ciudadanos en una cultura de la violencia. De esta manera, desde hace más de una década los temas de formación en democracia, derechos humanos, ciudadanía y convivencia han sido incluidos con más frecuencia en el currículo escolar.

Para ilustrar, tenemos de referencia la normatividad en torno a este tipo de contenidos como lo referencia Salguero (2005) por ejemplo en la ley de educación 115 de 1994 que en su artículo 14 expresa que en la educación preescolar básica y media del nivel oficial y privado debe contemplarse una educación para la justicia, la paz, la democracia, solidaridad y la formación en valores. Aspectos que se deben formar como parte de unos contenidos transversales a todo el plan de estudios y no como una asignatura especifica.

Por otro lado, está la resolución 1600/ 94 que establece la formación en democracia en todos los niveles educativos. También en la normatividad se cuenta con el programa de educación para el ejercicio de los derechos humanos (Eduderechos) iniciativa que surge en el año 2003 como parte del Ministerio de Educación Nacional. Además del componente de construcción de ciudadanía articulado en el programa de educación para la sexualidad y construcción de ciudadanía (PESCC).

Todos los programas mencionados se contemplan como transversales al plan de estudios y no como asignaturas específicas.

Actualmente con los procesos de negociación de la paz con las FARC en la Habana, Cuba, el gobierno Nacional ha dispuesto como parte de una fase de posconflicto un entramado normativo para enfatizar el valor de la educación en la construcción de una cultura de paz. Por consiguiente, ha establecido mediante la ley 1732 del 1 de septiembre de 2014 el desarrollo de la cátedra de la paz en todas las instituciones educativas del país. La cátedra se erige como una asignatura independiente y de obligatorio cumplimiento.

En la propuesta de contenidos de la cátedra para la paz se propone reflexionar sobre temas como la igualdad, respeto a los derechos, participación ciudadana, justicia social y economía equitativa.
De esta iniciativa cabe resaltar la importancia de que el gobierno reafirme el papel crucial que va a jugar la educación en la construcción de una cultura de paz. Sin embargo, son varias las cuestiones que suscita esta propuesta de cátedra para la paz, como: ¿qué concepción de ser humano para la paz pretende el gobierno formar desde la presente cátedra?; ¿qué concepción de paz se pretende construir? Y ¿quiénes serán los encargados de posibilitar la reflexión en torno a la paz? Aspectos que son importantes tener en cuenta en la creación de unas condiciones necesarias para una efectiva construcción de cultura de paz que supere las ideas pasivas sobre la paz para una comprensión de una educación para la paz compleja y que invita a replantearse muchos aspectos del sistema educativo tradicional.

\section{Los retos que la educación para la paz le plantea a la escuela}

La educación para la paz sin fomenta la reflexión con relación a la redefinición de aspectos como la estructura de la escuela, las relaciones que se establecen en la comunidad educativa, los modelos educativos entre otros aspectos, que le plantean varios retos a la escuela, como los siguientes:

1. La flexibilización del fenómeno de la paz en el currículo escolar, en la cual no sea vista como una asignatura independiente sino como un aspecto que atraviesa toda la estructura y vida escolar, es decir, como un principio Educativo y no como una asignatura más.

2. Fomentar la paz desde una pedagogía de la crítica y socio- afectiva, donde se rescate el valor de la expresión de las emociones como parte de la comprensión y acercamiento entre los seres humanos. Por lo general, en las escuelas se fomenta el control de las emociones, bajo la premisa general de la racionalidad. Elemento muy asociado a la fragmentación del ser humano entre el sentir y el pensar, asumiéndose la expresión del sentir como algo negativo. Por lo tanto, no se trata de menguar el valor de las emociones sino potencializar su valor para las relaciones humanas y posibilitando que estas se transformen de una manera positiva que permite la empatía, la compasión y la comunicación no violenta con el otro.

3. No limitar la educación para la paz a un proceso exclusivamente cognitivo, de aprendizaje sobre contenidos de paz, la paz va más allá del discurso y debe comprenderse como algo real, ligado a las experiencias y realidades cotidianas. La paz hecha cuerpo y hecha vivencia, esto posibilita que la paz no se vea como algo lejano y abstracto sino como un "posible" que todos y todas podemos construir desde nuestras distintas formas de habitar los territorios.

4. Posibilitar una educación para la paz centrada en la persona y el fomento de capacidades que le permitan a cada persona hacer y ser. Una educación centrada en la persona implica reconocer la 
diversidad, las historias de vida distintas y la paz como un proceso de trasformación personal que posteriormente se refleja en transformaciones pequeñas, en microespacios como la escuela, la familia y el barrio.

5. Redefinir la estructura jerárquica y poco democrática de la escuela, donde las relaciones entre los docentes y estudiantes sigue desarrollándose bajo relaciones de poder y verticales, donde el docente es quien posee el saber absoluto y el estudiante es quien espera pasivamente incorporar dichos saberes. La educación para la paz implica reconocer los saberes tanto de estudiantes como docentes en torno a la paz, el fomento de relaciones horizontales y el reconocimiento del otro. Si hablamos de algo contrario a la educación para la paz es educar desde la imposición.

6. Reflexionar en torno a los medios y fines en torno a la educación para la paz, no se puede concebir una educación para la paz fundamentada en lógicas de violencia. Prácticas escolares donde se impongan los saberes de uno sobre otro, o la homogenización de las personas son prácticas que en lugar de educar para la paz propician la justificación de lógicas violentas. En tal sentido, una educación para la paz debe posibilitar la crítica, la creatividad, la incertidumbre y la liberación.

Los retos que tiene la escuela en el desarrollo de una educación para la paz implica reflexiones estructurales y epistemológicas, que desde hace muchos años se vienen proponiendo desde las pedagogías libertarias y que el contexto educativo colombiano debe recorrer para pensar que es posible que la educación sea un medio fundamental para la construcción de una cultura de paz.

\section{Conclusiones}

1. La construcción de una cultura de paz debe ir acompañada de dos factores principales: la educación y la praxis social. Una educación que fomente la confianza, la crítica, la cooperación, el reconocimiento de la diversidad y la emancipación como motor movilice la praxis social en torno a la construcción de paz, con la formación de niños, niñas y jóvenes conocedores de sus realidades y dispuestos a hacer posible un país donde cada uno pueda tener una vida digna.

2. La educación para la paz debe tener como propósito desaprender las lógicas de una cultura de la violencia basada en la competencia, la falta de solidaridad, el rechazo a la diversidad y los valores militaristas para posibilitar la construcción de una cultura de paz que propenda por el aprendizaje cooperativo, la empatía, la compasión y la emancipación.

3. La educación para la paz contempla replantearse no solo cuestiones de forma como la inclusión en los currículos o la definición de los contenidos a desarrollar sino que de fondo implica reflexiones pedagógicas y epistemológicas en torno al concepción de cómo se entiende un sujeto de paz, que tipo de paz se concibe y que modelo pedagógico es necesario para posibilitar una educación para la paz.

\section{Referencias Bibliográficas}

Jiménez, M. (2011). La paz en la educación, Bogotá. Editorial: Observatorio para la paz.

Ley 1732. Portal virtual actualícese.com. Colombia, 1 de septiembre de 2014.

Ministerio de Educación Nacional. Programa de educación para el ejercicio de los Derechos Humanos. Modulo I. recuperado de: http://www.mineducacion.gov.co/1621/w3-article-241325.html

Salguero, J. M. (2005). Educar para la paz: el caso de un país dominado por la violencia: Colombia. Tesis (Doctor en ciencias de la Educación). Madrid: Universidad Complutense de Madrid. Facultad de Educación. Departamento de Didáctica y Organización Escolar. (Versión electrónica). Disponible en: http://eprints.ucm.es/tesis/edu/ucm-t27290.pdf, p. 278. 